O. Burkhardt ${ }^{1}$

E. Straube ${ }^{2}$

T. Welte ${ }^{1}$

\section{Klinisches Bild, Diagnostik und Therapie der Chlamydien-Pneumonie}

Clinical Picture, Diagnosis and Treatment of Chlamydia Pneumonia

\section{Einleitung}

In den letzten Jahren wurde mehrfach über einen kontinuierlichen Wandel im Erregerspektrum der ambulant erworbenen Pneumonie berichtet. Hierbei zeigte sich der allgemeine Trend einer relativen Zunahme so genannter atypischer Pneumonieerreger bei gleichzeitigem Rückgang grampositiver Mikroorganismen [1 -8]. Zu diesen atypischen bzw. intrazellulär agierenden Bakterien gehören auch die Chlamydien. In epidemiologischen Studien wurde in bis zu 15\% aller erfassten Pneumoniefälle Chlamydia pneumoniae identifiziert $[1-5,9,10]$. Gleichzeitig gelang jedoch häufig der Nachweis eines zweiten oder dritten Erregers im Sinne einer Mischinfektion, so dass die Frage nach der pathogenetischen Bedeutung von Chlamydia pneumoniae für die Pneumonie zum Teil sehr kontrovers diskutiert wurde [11 - 13]. Das derzeitige Hauptproblem und der Schwerpunkt in der Chlamydienforschung betrifft die Diagnostik dieser obligat intrazellulären, gramnegativen Bakterien [14]. Trotz einer Vielzahl von Nachweismethoden ist zur Zeit keine Technik in der Lage, einen schnellen und zugleich verlässlichen Erregernachweis zu erbringen. Zudem fehlt noch jegliche Standardisierung. Zur Therapie von Chlamydieninfektionen insbesondere der Pneumonie stehen sehr potente Antibiotika wie die Makrolide und das Azalid Azithromycin, das Ketolid Telithromycin, das Tetracyclin Doxycyclin und die Fluorochinolone der neuen Generation zur Verfügung.
Erreger

Chlamydien gehören zur Familie der Chlamydiaceae [15 - 17]. Es handelt sich um gramnegative Bakterien, die unbeweglich sind, deren Zellwand charaktischerweise keine Peptidoglykanschicht enthält und die obligat intrazellulär leben. Gemeinsamkeit aller Chlamydien ist ihr komplexer Reproduktionszyklus, der zwei Erregerstadien umfasst. Die Infektionsform ist das extrazelluläre Elementarkörperchen, das sich an die Zielzellmembran heftet und mittels Phagozytose in die Zelle eindringt. Nach dem Eindringen umgibt es sich mit einer Einschlussmembran und bildet sich in dieser Vakuole zu dem nicht infektiösen Retikularkörperchen um. Dieses verdoppelt sich intrazellulär durch binäre Zellteilung. Innerhalb von 18 bis 24 Stunden kondensieren die Retikularkörperchen wiederum zu Elementarkörperchen, die dann nach der Freisetzung einen neuen Infektionszyklus beginnen. Chlamydien können kein ATP synthetisieren und sind deshalb auf die intrazelluläre Vermehrung in Wirtszellen und deren ATPSynthese angewiesen. Zur Familie der Chlamydiaceae gehören neben den drei klassischen humanpathogenen Arten Chlamydia trachomatis, Chlamydia psittaci und Chlamydia pneumoniae (neuerdings in Chlamydophila pneumoniae umbenannt) noch weitere Chlamydienarten wie Chlamydia pecorum, Chlamydia muridarum, Chlamydia suis, Chlamydia abortus, Chlamydia caviae und Chlamydia felis, deren Rolle in der Humanpathologie derzeit noch nicht geklärt ist [16-18]. Darüber hinaus gelang kürzlich der Nachweis chlamydienähnlicher Bakterien als Endosymbionten frei lebender Amöben (Neochlamydia hartmanellae und Parachlamydia acanthamoebae) [19-21] sowie als Kontaminanten einer Zellkultur (Simkania negevensis) [22]. Es liegen

Serienherausgeber: S. Ewig, T. Schaberg

Institutsangaben

${ }^{1}$ Abteilung für Pneumologie und Internistische Intensivtherapie (Leiter: Priv.-Doz. Dr. med. T. Welte), Medizinische Fakultät der Otto-von-Guericke-Universität Magdeburg ${ }^{2}$ Institut für Medizinische Mikrobiologie (Leiter: Prof. Dr. med. E. Straube), Friedrich-Schiller-Universität Jena

Korrespondenzadresse

Priv.-Doz. Dr. med. T. Welte · Otto-von-Guericke-Universität Magdeburg, Medizinische Fakultät . Abteilung für Pneumologie und Internistische Intensivtherapie · Leipziger Straße 44 · 39120 Magdeburg E-mail: tobias.welte@medizin.uni-magdeburg.de 
Hinweise vor, dass diese so genannten Umweltchlamydien Mitverursacher von Atemwegserkrankungen sein sollen. Auf der Basis einer 16S- und 23S-rRNA-Analyse teilen Everett und Mitarb. (1999) die Ordnung der Chlamydiales in 4 Familien ein: die Chlamydiaceae, die Simkaniaceae, die Parachlamydiaciae und die Waddliaceae (Tab. 1) [18].

Alle 3 bekannten humanpathogenen Chlamydienarten sind in der Lage eine Pneumonie beim Menschen auszulösen. Chlamydia trachomatis ist für eine Pneumonie beim Neugeborenen und Chlamydia psittaci für die in Deutschland meldepflichtige Ornithose bzw. Psittakose verantwortlich. Am häufigsten wird aber weltweit Chlamydia pneumoniae mit der ambulant erworbenen Pneumonie in Verbindung gebracht [23-29] und auch andere respiratorische Krankheiten wie die Sinusitis, die Pharyngitis und die akute Bronchitis vermag der Erreger auszulösen [30]. Eine Assoziation mit der chronischen Bronchitis [31,32] und dem Asthma bronchiale $[33,34]$ ist bekannt. Darüber hinaus wird über eine nicht unwichtige Rolle des Erregers in der Pathogenese schwerer nicht respiratorischer Erkrankungen wie der Arteriosklerose [35-38] oder der Alzheimer-Demenz [39,40] diskutiert. Erstmalig wurde Chlamydia-pneumoniae 1965 von der Konjunktiva eines taiwanesischen Kindes isoliert. Die Assoziation mit der Pneumonie erfolgte 20 Jahre später durch Saikku u. Mitarb. [41]. Obwohl tierische Chlamydia pneumoniae-Biovare vom Pferd (Biovar Equine) oder vom Koalabären (Biovar Koala) isoliert wurden [42], stellt der Mensch das alleinige Erregerreservoir für den humanpathogenen Stamm (Biovar TWAR) dar. Der Erreger selbst wird auf aerogenem Weg und durch Speichelkontakt von Mensch zu Mensch übertragen.

Als Erregerreservoir von Chlamydia psittaci, dem Erreger der Ornithose, sind vor allem Vögel (Papageien, Truthühner, Enten, Tauben, u.a.) aber auch andere Haustierrassen (Hunde, Katzen, Ziegen, Schafe, Kühe, u. a.) zu nennen $[16,17]$. Stämme von Vögeln, insbesondere Psittaciden (papageienartige) weisen aber eine höhere Humanpathogenität auf als die Stämme anderer Tierarten. Besonders Vögel von Tierfarmen (z. B. Enten oder Tauben) spielen für den Menschen als Infektionsquelle die wichtigste Rolle. Chlamydia psittaci kommt bei infizierte Tieren in respiratorischen Sekreten, Exkrementen und Federn vor und kann bei Raumtemperatur selbst bei Austrocknung ca. 4 Wochen infektiös bleiben. Die Übertragung auf den Menschen erfolgt aerogen, aber auch durch direkte Berührung der Vögel, selten durch Vogelbiss und noch seltener von Mensch zu Mensch.

\section{Inzidenz}

Obwohl die Inzidenz von Jahr zu Jahr und innerhalb geografischer Regionen variiert, wird geschätzt, dass 5-15\% der ambulant erworbenen Pneumonien durch Chlamydia pneumoniae verursacht werden $[1-5,9,10,23-29]$. Gleichzeitig gelang mehreren Untersuchern gelegentlich der Nachweis eines zweiten oder dritten Erregers im Sinne einer Mischinfektion, so dass die kontrovers diskutierte Frage nach der pathogenetischen Bedeutung Chlamydia pneumoniaes für die Pneumonie unbeantwortet blieb [11-13]. Ob Chlamydia pneumoniae allein in der Lage ist, eine pneumonische Entzündung auszulösen und zu erhalten oder ob der Erreger über eine initiale Schädigung des respiratori-
Tab. 1 Neue Taxonomie der Ordnung Chlamydiales nach Everett u. Mitarb. (1999) [18]

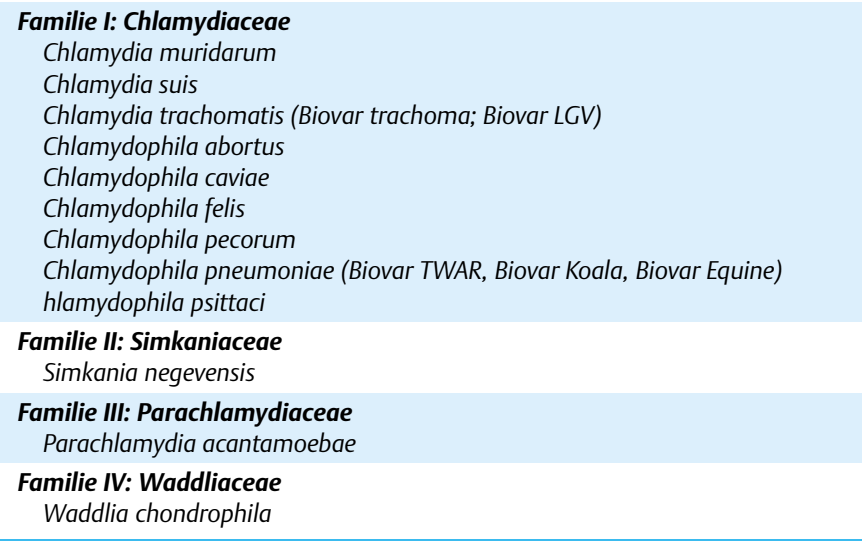

schen Epithels eine bakterielle Superinfektion fördert, beispielsweise durch Streptokokkus pneumoniae, einem häufig koinzidenten Keim der Chlamydia-pneumoniae-Pneumonie, müssen künftige Untersuchungen aufzeigen.

Über die Rolle von Chlamydia pneumoniae als opportunistischem Keim bei immunsupprimierten Patienten ist sehr wenig bekannt. Ob dieser Personenkreis einer erhöhten Gefahr für Chlamydia-pneumoniae-Infektionen ausgesetzt ist oder ob die Pneumonie innerhalb dieses Kollektivs schwerer verläuft als bei immunkompetenten Menschen muss durch weitere Untersuchungen abgeklärt werden. Erwiesen scheint aber, dass Patienten mit einer chronischen Erkrankung wie der COPD häufiger an einer Pneumonie durch den Erreger erkranken als gesunde $[31,32]$.

Ein anderes Problem stellt die Tatsache dar, dass die meisten der vorhandenen Daten über die Inzidenz von Chlamydieninfektionen durch die Auswertung serologischer Untersuchungen gewonnen wurden. Nur in Ausnahmefällen konnte die serologische Diagnose durch den kulturellen Nachweis von Chlamydia pneumoniae bestätigt werden. Diese serologischen Untersuchungen zeigen aber auch, dass Chlamydia pneumoniae ein weltweit häufiges Pathogen ist und dass die meisten Menschen mindestens einmal in ihrem Leben mit dem Erreger in Kontakt getreten sind. So finden sich bei ca. $50 \%$ aller Personen über 20 Jahre und bei ca. $75 \%$ der älteren Bevölkerung (>60 Jahre) im Serum deutlich messbare Antikörperspiegel gegenüber Chlamydia pneumoniae [43-47]. Dieser Aspekt der steigenden Seroprävalenz mit dem Alter könnte andererseits auch für eine lebenslange Persistenz des Erregers in menschlichen Gewebszellen und damit für eine chronisch-latente Infektion durch Chlamydia pneumoniae sprechen. Auch dieser Punkt erfordert weitere Untersuchungen.

Die im Rahmen der Ornithose auftretende Chlamydia psittaciPneumonie wird ebenfalls weltweit registriert $[16,17]$. In Deutschland werden jährlich ca. 100 Neuerkrankungsfälle gemeldet. Betroffen ist häufig Personal von Geflügelfarmen oder Vogelhandlungen. 
Die Inkubationszeit der Chlamydia pneumoniae-Pneumonie ist länger als bei anderen respiratorischen Erregern und kann vom Kontakt bis zum Ausbruch der Pneumonie 4 Wochen betragen [15-17]. Im Gegensatz zur klassischen bakteriellen Pneumonie findet sich ein protrahierter Krankheitsbeginn, der vor allem durch das Auftreten von Allgemeinsymptomen wie Kopf- und Gliederschmerzen und Fieber ohne Schüttelfrost charakterisiert ist. Es besteht meist ein trockener Reizhusten mit spärlichem oder fehlendem Auswurf sowie eine relative Bradykardie $[11,26,29]$. Darüber hinaus stellt sich ein Missverhältnis zwischen geringem Auskultationsbefund und relativ deutlichem Röntgenbefund dar. Die Ursache hierfür liegt im bevorzugten Befall der zentralen Lungenabschnitte durch den atypischen Erreger. Ein negativer Perkussions- und Auskultationsbefund schließt demnach eine Chlamydia pneumoniae-Pneumonie nicht aus. Im Zweifelsfall sollte daher immer eine röntgenologische Untersuchung der Lungen erfolgen. Laborchemisch finden sich häufig normale oder erniedrigte Leukozytenzahlen mit einer relativen Lymphozytose bei mäßig erhöhtem C-reaktiven Protein.

Hinsichtlich des Verlaufs bzw. der Prognose der Chlamydia pneumoniae-Pneumonie ist zu sagen, dass diese meist mild verläuft und in nur sehr seltenen Fällen eine Krankenhausbehandlung erfordert $[11,26,29]$. Typischerweise bilden sich die Krankheitssymptome unter Antibiotikatherapie dann aber nur sehr langsam zurück, so dass der charakteristische Husten und die Störung des Allgemeinbefindens noch mehrere Wochen über das Therapieende hinaus persistieren können. Findet sich dagegen das Bild einer Mischinfektion, ggf. mit Streptococcus pneumoniae als zweitem Erreger, ist der Patient schwerwiegender erkrankt [48-51]. Dies führt wiederum nicht selten aufgrund einer respiratorischen Insuffizienz zur Beatmungs- und Intensivüberwachungspflichtigkeit des Erkrankten. Die häufigste Todesursache stellt hierbei die Pneumokokkensepsis dar.

Der Krankheitsverlauf der durch Chlamydia psittaci verursachten Ornithose ist recht variabel [52 - 54]. Der Schweregrad reicht von leichten Erkrankungen bis zu tödlich verlaufenden systemischen Infektionen, bei denen die respiratorischen Symptome im Vordergrund stehen. Die Ornithose beginnt häufig plötzlich mit hohem Fieber und Schüttelfrost, wobei das Fieber in Form eines Febris continua bei relativer Bradykardie verläuft. Weitere charakteristische Beschwerden sind Kopfschmerzen, abdominelle Beschwerden, Myalgien und Lichtscheu sowie trockener Husten mit mäßig produktivem, gelegentlich hämorrhagischem Auswurf. Selten kommt es auch zum Auftreten so genannter „Horder's spots“, einem Exanthem, das den Typhusroseolen ähnelt. Bei schweren Krankheitsverläufen können eine Hepatosplenomegalie, Verwirrtheitszustände, Delirien, Stupor, Hypoxie und Zyanose auftreten. Aber auch Erkrankungsbilder wie Endokarditis, Myokarditis, Perikarditis und Enzephalitis sind möglich.

\section{Diagnostik}

Die Diagnostik der Pneumonie durch Chlamydia pneumoniae stellt nach wie vor eine große Herausforderung dar und gehört mit zu den Hauptschwerpunkten in der Erforschung des Erregers. Generell existieren verschiedene praktizierte Möglichkeiten des Erregernachweises, entweder direkt durch die Kultur, den Antigennachweis oder die PCR oder indirekt über die Bestimmung von Chlamydia pneumoniae-Antikörpern im Serum des Patienten [15-17]. Jede dieser Methoden weist eigene, zu beachtende Besonderheiten auf und die Ergebnisse variieren von Labor zu Labor, so dass sie teilweise nur sehr schwer zu interpretieren sind. Letztgenanntes unterstreicht die Notwendigkeit einer Standardisierung der verwendeten Verfahren. Dieser Aufgabe hat sich eine international gebildete Arbeitsgruppe namhafter Chlamydienforscher angenommen, welche im Auftrag des Centers for Disease Control and Prevention (USA) und des Laboratory Centre for Disease Control (Kanada) erste Empfehlungen für eine standardisierte Chlamydiendiagnostik schriftlich festhielten [14].

Der kulturelle Nachweis gilt als der Goldstandard zur Diagnostik von Chlamydieninfektionen, spielt aber aus mehreren Gründen für die Akutdiagnose der Chlamydien-Pneumonie nur eine untergeordnete Rolle. Im Gegensatz zu anderen Bakterien gelingt die Anzucht von Chlamydia pneumoniae, als obligat intrazellulärem Erreger, nur in der Zellkultur auf speziellen eukaryonten Zelllinien, wie der HL- oder der HEp-2-Zelllinie [55-57]. Diese müssen vor der notwendigen Aufzentrifugation des Atemwegsmaterials (bronchoalveoläre Lavage, verdünntes Tracheobronchialsekret) mit Polyethylenglycol (PEG), Trypsin und Diethylaminoethyldextran vorbehandelt werden. Die Anlage dieser Kulturen über mehrere Passagen kann deshalb nur in Speziallaboren erfolgen. Dies bedeutet aber wiederum lange Wege und damit einen sehr großen Zeitaufwand bis zur Erregerdiagnose, der aufgrund der Akutität des Krankheitsbildes ungerechtfertigt erscheint. Zudem ist die Kultur nur in sehr wenigen Fällen erfolgreich und die Spezifität ihrer Ergebnisse hängt sehr von der mikroskopischen Erfahrung des auswertenden Laboranten $a b$, Chlamydieneinschlüsse von Färbeartefakten zu unterscheiden.

Im Gegensatz zur Pneumokokken- und Legionellenpneumonie, wo für die Erregerschnelldiagnostik spezielle hochsensitive und hochspezifische Bedside-Tests zum Nachweis der entsprechenden Antigene im Urin vorliegen, findet sich zur Diagnostik der Chlamydia pneumoniae-Pneumonie derzeit kein vergleichbarer Antigenschnelltest. Ein im Handel erhältlicher gruppenspezifischer Antigen-ELISA dient in einigen Laboren dem Nachweis von Chlamydia trachomatis, vor allem bei Verdacht auf Infektionen des Urogenitaltraktes. Aufgrund seiner geringen Sensitivität und Spezifität ist dieser für die Chlamydia pneumoniae-Diagnostik nicht zu empfehlen. Ein anderer Antigen-Test, der direkte Immunfluoreszenz-Antikörper-Test (DFA) verwendet für die Antigen-Detektion Chlamydia pneumoniae-spezifische FITC-markierte monoklonale Antikörper. Zwar eignet er sich hervorragend zum Nachweis des Erregers in der Zellkultur, für die Begutachtung von Direktpräparaten des Atemwegssekretes gilt der DFA dagegen als zu insensitiv $[58,59]$.

Aufgrund der genannten Unzulänglichkeiten von kulturellem Erregernachweis und nicht kulturellem Antigennachweis wird die Diagnose einer Chlamydia pneumoniae-Pneumonie zum jetzigen Zeitpunkt fast ausnahmslos serologisch durch den Nachweis spezifischer Antikörper gestellt [14,15-17]. Dies ist aber aus 
mehreren Gründen nicht ganz unproblematisch. Schwierigkeiten für die serologischen Untersuchungen ergeben sich beispielsweise aus dem seltenen Erhalt gepaarter Serumproben, dem Fehlen standardisierter Testverfahren und dem Mangel an hochqualitativen Testreagenzien. Auch die bereits angesprochene hohe Seroprävalenz in der erwachsenen Bevölkerung muss bei der Testbewertung berücksichtigt werden. Darüber hinaus scheint eine gewisse Kreuzreaktivität zu anderen Infektionserregern wie Mykoplasma pneumoniae oder dem Parvovirus B19 zu bestehen $[60,61]$.

Das Verstehen und die Auswertung der serologischen Ergebnisse erfordert die Kenntnis der Antikörperkinetik von Chlamydia pneumoniae [15]. Die Erstinfektion mit dem Erreger ist durch eine frühe IgM-Antwort innerhalb von 2 bis 3 Wochen, eine verzögerte IgG-Antwort innerhalb von 6 bis 8 Wochen und eine schwache oder fehlende IgA-Antwort charakterisiert. Nach der akuten Infektion verschwinden die IgM-Antikörper gewöhnlich nach 2 bis 4 Monaten. Hohe IgG-Antikörpertiter können dagegen bei Fehlen klinischer Zeichen persistieren, während IgA-Antikörper dahin tendieren, schnell zu verschwinden. Bei der Chlamydia pneumoniae-Reinfektion fehlt charakteristischerweise die IgM-Antwort, bei einem sofortigen IgG- und IgA-Antikörperanstieg. In Anbetracht der Tatsache, dass bei vielen Menschen die meist asymptomatische Erstinfektion mit dem Erreger bereits vor dem 20. Lebensjahr erfolgte, lässt sich die Schwierigkeit abschätzen, eine akute von einer ehemals stattgefundenen Infektion abzugrenzen. Hierfür liegen Bewertungskriterien vor, welche bei der Auswertung serologischer Ergebnisse unbedingt zu beachten sind [14]. Eine akute Infektion mit Chlamydia pneumoniae findet sich bei einem IgM-Titer von 16 oder einem vierfachen IgG-Titeranstieg, ein Verdacht auf eine akute Infektion bei einem IgG-Titer von 512 und eine zurückliegende Infektion bei einem IgG-Titer von 16. Die Bedeutung der IgA-Antikörper ist in diesem Zusammenhang noch unklar, wird aber häufig als Zeichen persistierender Infektionen angesehen. Es empfiehlt sich also, sowohl IgG- als auch IgM- und IgA-Antikörper bei allen Patienten zu bestimmen, bei denen der Verdacht auf eine Chlamydia pneumoniae-Pneumonie besteht.

Für die serologischen Untersuchungen stehen mehrere Testverfahren (KBR, IFA, EIA, ELISA, Immunoblot) zur Verfügung. Je nachdem welches Chlamydien-Antigen (LPS, Elementarkörperchen, MOMP-Protein) für die Antikörperdetektion verwendet wird, unterscheidet man gattungs- bzw. gruppenspezifische von spezies- und serospezifischen Testen.

Die veraltete Chlamydien-Komplementbindungsreaktion verwendet ein angereichertes LPS-Antigen zur Erkennung von Gruppen-Antikörpern, kann nicht zwischen den einzelnen Chlamydienarten differenzieren und zeigt sogar Kreuzreaktionen zu einigen Enterobakteriaciae. Sie ist technisch schwierig durchzuführen und für die Detektion einer Reinfektion nicht sensitiv genug [62-64].

Bei den Indirekten Immunfluoreszenz-Assays (IFA) unterscheidet man zwei Arten. Der erste IFA verwendet infizierte Zellen, welche das komplette Einschlusskörperchen als Substrat expremieren. Retikularkörperchen, aus denen die Einschlusskörperchen zusammengesetzt sind, weisen aber nur gattungsspezifi- sche Epitope auf und erlauben deshalb keinen differenzierten Nachweis einer chlamydienspezifischen Antikörperreaktion [63]. Auch dieser Test ist veraltet und spielt für die Diagnostik der Chlamydia pneumoniae-Infektion keine Rolle mehr.

Der andere IFA, der Mikroimmunfluoreszenz Assay (MIF), gilt noch immer als Referenzmethode in der artspezifischen Serologie [65 - 72]. Beim MIF handelt es sich um einen sehr aufwendigen, subjektiv auszuwertenden Test, der viel Erfahrung erfordert und deshalb nur in Speziallaboren durchgeführt wird. Als Antigen dienen hierbei gereinigte formalinfixierte Elementarkörperchen, die nach Entfernung des gattungsspezifischen LPS-Antigens einzelne spezies- und serovarspezifische Chlamydien-Antikörper erkennen. Elementarkörperchen aller Chlamydienspezies und -serotypen können nebeneinander vereint oder als separate Substrate für die Antikörper-Erkennung genutzt werden. Neben der Trennung einzelner Chlamydienarten in einer Serumprobe kann der MIF aber auch die Antikörperklassen IgM, IgG und IgA voneinander unterscheiden, ist somit für die Beurteilung des aktuellen Infektstatus (akut oder chronisch) sehr hilfreich. Das Hauptproblem neben der technischen Komplexität des Testes und der subjektiven Interpretation der Testergebnisse stellt aber die fehlende Standardisierung sowohl bei den Testreagenzien als auch bei den diagnostischen Kriterien dar, so dass sich die Ergebnisse noch immer von Labor zu Labor unterscheiden. Die Expertengruppe der CDCP und der LCDC sprach Empfehlungen für den MIF aus (Tab. 2) [14].

Tab. 2 Empfehlungen für den MIF nach Dowell u. Mitarb. (2001) [14]

\begin{tabular}{|c|c|}
\hline Antigen: & $\begin{array}{l}\text { - Renografin-gereinigte Elementarkörperchen der drei } \\
\text { Spezies in PBS mit 0,02\% Formalin und 0,5\% Dotter- } \\
\text { sackflüssigkeit in feinsten Spots nebeneinander in } \\
\text { einem Reaktionsfeld auf Objektträger } \\
\text { - Fixierung mit Aceton }\end{array}$ \\
\hline Serum: & - Serumpaare mit 4 bis 8 Wochen Entnahmeabstand \\
\hline Durchführung: & $\begin{array}{l}\text { - Verdünnung } 1: 8,1: 16,1: 32 \text {, usw. } \\
\text { - } \text { Absorption von Rheumafaktoren bei Messung von IgM } \\
\text { und IgA } \\
\text { - Gegenfärbung der mit FITC-Konjugat entwickelten } \\
\text { Felder mit Evan's blue }(0,05 \%) \text { oder Rhodamin- } \\
\text { konjugiertem BSA }(1 / 15)\end{array}$ \\
\hline Ablesung: & - Objektiv 40fach, achromat, Okular 10fach \\
\hline Bewertung: & $\begin{array}{l}\text { - akute Infektion: IgM-Titer = 16, vierfacher IgG-Titer- } \\
\quad \text { Anstieg } \\
\text { - akute Infektion möglich: IgG-Titer }=512 \\
\text { - zurückliegende Infektion: IgG-Titer = } 16\end{array}$ \\
\hline Kontrollen: & $\begin{array}{l}\text { - positive/negative Serumkontrollen } \\
\text { - optimale Konjugatverdünnung mit hochtitrigem Serum } \\
\text { festlegen } \\
\text { - Konjugat aliquotieren, bei }-20^{\circ} \mathrm{C} \text { aufbewahren } \\
\text { - Ableser erfahren, aber ohne Erwartungswerte }\end{array}$ \\
\hline
\end{tabular}

Die kostengünstigeren Alternativen zum Mikroimmunfluoreszenz Assay stellen in der heutigen Zeit die teilweise automatisierten, speziesspezifischen ELISA-(enzyme-linked immunosorbent assay) und EIA-(enzyme immunoassay) Testverfahren dar. Im Gegensatz zum MIF sind beide Methoden standardisierter und die Auswertung ihrer Ergebnisse erfolgt quantitativ. Sie un- 
terliegen weniger dem subjektiven Einfluss des jeweiligen Untersuchers und eignen sich damit besser für die Routinediagnostik des Erregers. Als Antigene dienen aufgereinigte Bestandteile der so genannten MOMP-Proteine (major outer membrane protein), den Hauptmembranproteinen in der chlamydialen Zellwand, die als speziesspezifisch zu charakterisieren sind. Darüber hinaus gelingt durch den getrennten Nachweis von IgM-, IgG- und IgAAntikörpern gegen Chlamydia pneumoniae, analog zum MIF, eine Abklärung des Infektionsstatus und des Therapieverlaufes. Trotz des Mangels an publizierten Daten liegen erste Hinweise für eine ausreichende Sensitivität und Spezifität einiger kommerziell erhältlicher ELISAs und EIAs bereits vor [71,72]. So verglichen Hermann u. Mitarb. (2002) [72] kürzlich im Rahmen von Untersuchungen zur Seroprävalenz von Chlamydia pneumoniaespezifischem IgG bei 80 gesunden Probanden sieben im Handel erhältliche Testkits (4 ELISAs, 3 EIAs) mit vier kommerziell erhältlichen MIFs. Hierbei zeigte sich eine gute Korrelation der Ergebnisse sowohl bei den 4 untersuchten MIFs (range 83-99\%) als auch zwischen den MIFs und den verschiedenen ELISAs und EIAs (range 78-98\%). Die Spezifität aller speziesspezifischen Tests variierte von 95 bis $98 \%$, die Sensitivität von 58 bis $100 \%$. Trotz der erkennbaren Schwankungen waren Sensitivität und Spezifität der ELISAs und EIAs mit denen der MIFs vergleichbar. Ein anderes, nicht unwesentliches Problem offenbarte sich jedoch bei der Bestimmung der IgG-Antikörper-Titer durch die unterschiedlichen Testverfahren. Während die mittels der 4 MIFs gemessenen IgG-Titer in ihren Werten gut übereinstimmten, waren alle verwendeten ELISAs und EIAs nur mäßig in der Lage, niedrige Antikörperspiegel zu erkennen. Hieraus leiteten die Autoren ab, dass der MIF auch weiterhin als die empfindlichste Methode zur Chlamydia pneumoniae-Antikörperdetektion anzusehen ist. Der beschriebene ELISA- und EIA-Engpass muss dagegen durch weiterführende Untersuchungen abgeklärt werden.

Ein anderer, noch zu erwähnender serologischer Test ist der Immunoblot. Er dient bisher vornehmlich der serologischen Sicherung einer Borrelien-Infektion [73]. Abgewandelte, für Chlamydien spezifische, aber nicht standardisierte Immunoblot-Verfahren können aufgrund ihrer methodischen Komplexität nur in Speziallaboren durchgeführt werden $[72,74]$.

In Anbetracht der Tatsache, dass die serologischen Untersuchungen eine Pneumonie durch Chlamydia pneumoniae allenfalls retrospektiv anzeigen, beruht die Hoffnung einer schnelleren Diagnosestellung auf den molekularbiologischen Methoden. Mit Hilfe der PCR, als bekanntestem molekularbiologischen Verfahren, kann Chlamydia pneumoniae innerhalb von Stunden als pneumonieverursachender Keim identifiziert werden. Der Nukleinsäurenachweis des Erregers gelang in verschiedenen Atemwegsmaterialien wie der bronchoalveolären Lavave $[50,75]$, dem Sputum $[69,76]$ und in Nasopharyngealabstrichen $[69,77-79]$. Die meisten der hierbei verwendeten PCR-Varianten gelten aber als so genannte In-house-Methoden, so dass ihre Ergebnisse bislang nur schwer nachprüfbar sind. Trotz scheinbarer Spezifität und Sensitivität unterscheiden sie sich voneinander in mehreren Punkten, so in der Materialgewinnung und -verarbeitung, in der Auswahl des Primers, in der Nukleinsäureextraktion, in der Detektion des Amplifikationsproduktes und in der Identifikation falsch positiver bzw. falsch negativer Ergebnisse. Hieraus lässt sich die Notwendigkeit einer Standardisierung sowohl in der
PCR-Methodik als auch in der Bewertung der PCR-Befunde ableiten [14].

In neuerer Zeit sind auch in der Chlamydiendiagnostik die konventionellen PCR-Verfahren zunehmend von den so genannten Real-Time-PCRs abgelöst worden. Diese Techniken besitzen den Vorteil in kürzerer Zeit, d.h. innerhalb von 1 bis 2 Stunden, eine aussagekräftige Information über den Infektionserreger liefern zu können. Zudem gelingt mit ihnen eine Quantifizierung Chlamydia pneumoniaes im jeweiligen Untersuchungsmaterial. Die Evaluation mehrerer Real-Time-PCRs gegenüber konventionellen PCR-Techniken und der Chlamydienkultur ist in verschiedenen Atemwegssekreten erfolgt [80-85]. Alle publizierten Verfahren sind von den Autoren als hoch spezifisch und hoch sensitiv beschrieben worden.

Als Nachweismethode der Zukunft, auch in der Chlamydiendiagnostik, ist die Fluoreszenz In-situ-Hybridisierung (FISH) anzusehen [86]. Sie hat gegenüber anderen Techniken den Vorzug, gleichzeitig Visualisierung, Identifikation und Lokalisierung von einzelnen Mikroorganismen zu ermöglichen. Sie findet bisher Anwendung bei umfassenden mikrobiologischen Fragestellungen in der Umwelt- und Biotechnologie und wird aber zunehmend auch zu diagnostischen Zwecken eingesetzt. Da man mit dieser molekularbiologischen Methode nicht nur kultivierbare, sondern auch anspruchsvolle und schlecht kultivierbare Bakterien wie die Chlamydien detektieren kann, ist FISH insbesondere für die Analyse komplexer Habitate geeignet.

Die Diagnostik der Ornithose stützt sich zunächst auf das klinische Bild und eine genaue Anamneseerhebung mit der Frage nach Vogelkontakten. Der Nachweis von Chlamydia psittaci erfolgt analog zum Nachweis von Chlamydia pneumoniae, entweder durch die Kultur, die PCR oder über die Bestimmung von spezifischen Antikörpern im Serum des Patienten. Beim kulturellen Nachweis von Chlamydia psittaci muss angemerkt werden, dass die Vermehrung des Erregers wegen des Risikos schwerer Laborinfektionen nur in Laboratorien der Sicherheitsstufe III zugelassen ist.

\section{Therapie}

Die Initialtherapie der ambulant erworbenen Pneumonie erfolgt in der Regel ungezielt. Chlamydien, als häufige Pneumonieerreger, müssen hierbei berücksichtigt werden [87]. Als Chlamydienwirksam gelten Tetracycline, Makrolide, Azalide, Ketolide und die Fluorochinolone, d.h. Antibiotika, die über einen Eingriff in die bakterielle Protein- oder DNS-Synthese die Erreger abtöten oder deren Wachstum inhibieren. $\beta$-Laktam-Antibiotika sind unwirksam. Die Guidelines nationaler und internationaler Fachgesellschaften [88-90] empfehlen die Gabe der genannten Substanzen auch zur gezielten Therapie der Chlamydien-Pneumonie, wobei aber vor allem ältere Vertreter dieser Antibiotikaklassen wie das Doxycyclin, das Erythromycin oder das Ciprofloxacin in den Richtlinien erwähnt sind. Die Tatsache, dass in den letzten Jahren neue wirksamere und auch verträglichere Antibiotika mit verbesserter Pharmakokinetik wie das Clarithromycin, das Azithromycin, das Telithromycin und die neuen Atemwegsfluo- 
rochinolone entwickelt wurden, erfordert eine Überarbeitung der bisherigen Therapieempfehlungen.

Die Daten zur In-vitro-Aktivität chlamydienwirksamer Antibiotika, auf denen alle bekannten Therapie-Guidelines beruhen, wurden vornehmlich durch Empfindlichkeitsmessungen der Bakterien in der Zellkultur gewonnen. Ob diese Daten aber ohne weiteres auf die Effektivität dieser Substanzen bei der Behandlung der Chlamydien-Pneumonie übertragbar sind, ist ungewiss. In den meisten bisher veröffentlichten Behandlungsstudien wurde die Diagnose einer Chlamydien-Pneumonie serologisch gestellt. Die Ermittlung der bakteriologischen Wirksamkeit in vivo war damit weitgehend unmöglich. Auch Daten zur klinischen Wirksamkeit chlamydienwirksamer Antibiotika existieren nur vereinzelt, so dass weiterführende Untersuchungen zu diesem Sachverhalt erfolgen müssen.

\section{Makrolide}

Als wirksamste Substanzgruppe zur Behandlung einer Chlamydia pneumoniae-Pneumonie gelten die Makrolid-Antibiotika. Die Muttersubstanz, das Erythromycin, wurde jahrelang als First-line-Therapeutikum bei Pneumonie durch atypische Erreger eingesetzt. Zudem war es lange Zeit als einziges Makrolid in einer i.v. Formulierung erhältlich, so dass bei schweren atypischen Pneumonien nicht auf die Gabe von Erythromycin verzichtet werden konnte. Der Nachteil des Erythromycins besteht vor allem in der mäßigen gastrointestinalen Verträglichkeit nach oraler Gabe seiner Ester und in dem Auftreten von nicht unerheblichen Venenreizungen nach intravenöser Applikation. Die neueren Makrolide wie das Clarithromycin oder das Azalid Azithromycin sind wirksamer, nebenwirkungsärmer und weisen ein verbessertes pharmakokinetisches Profil auf. Typisch für alle Makrolide, aber am ausgeprägtesten für Azithromycin nachweisbar, ist die erhebliche Anreicherung in tiefen Kompartimenten, d. h. in phagozytierenden Zellen und im Gewebe. In der Regel finden sich hier höhere Substanzkonzentrationen als im Blut.

Die In-vitro-Aktivität gegenüber Chlamydia pneumoniae ist bei den Makroliden am höchsten für Clarithromycin mit MHK-Werten zwischen 0,004 bis 0,03 mg/L [91 - 96]. Die MHKs (Minimale Hemmkonzentrationen) des Azithromycins $(0,05-0,25 \mathrm{mg} / \mathrm{L})$ [91 - 94,97] entsprechen weitgehend den MHKs des Erythomycins $(0,008-0,25 \mathrm{mg} / \mathrm{L})[91-94,98-101]$.

Das aber über die In-vitro-Aktivität nicht in jedem Falle auf die bakterielle Wirksamkeit in vivo zurückgeschlossen werden kann, beweist die Studie von Block u. Mitarb. (1995) [102]. Hier wurden Kinder mit ambulant erworbener Chlamydia pneumoniaePneumonie entweder mit Erythromycin oder Clarithromycin behandelt. Trotz zehn- bis hundertfach höherer In-vitro-Aktivität des Clarithromycins war Erythromycin signifikant effektiver in der Eradikation von Chlamydia pneumoniae als Clarithromycin ( $86 \%$ versus $79 \%$ ). Eine Nachfolgestudie von Roblin und Hammerschlag (1998) [103] beschäftigte sich mit der bakteriologischen Wirksamkeit des Azithromycins. 26 Kinder und Erwachsene mit kulturell nachgewiesener Pneumonie durch Chlamydia pneumoniae wurden einer Behandlung mit Azithromycin zugeführt. Bei 7 der 26 Patienten war trotz klinischer Besserung die Chlamydienkultur nach dem Ende der Therapie weiterhin positiv, so dass von einer Erregerpersistenz ausgegangen werden musste. Ein Anhalt für eine Resistenzentwicklung unter Therapie ergab sich nicht. Dennoch fand sich nach Therapieende bei $2 \mathrm{~Pa}-$ tienten ein vierfacher MHK-Anstieg bei den entsprechenden klinischen Isolaten.

Sowohl vom Clarithromycin als auch vom Azithromycin sind bereits i.v. Lösungen erhältlich, so dass auch bei schweren Chlamydieninfektionen auf die Gabe des veralteten Erythromycins verzichtet werden kann. Die Gabe von Clarithromycin erfolgt je nach Schwere der Infektion oral oder intravenös in einer Dosierung von $2 \times 500 \mathrm{mg}$ täglich über 10 bis 14 Tage. Die übliche therapeutische Dosis von Azithromycin beträgt 1,5g. Diese Dosis wird über einen Zeitraum von 3 bis 5 Tagen appliziert. Wegen der lang andauernden Gewebsspiegel ist die Therapiedauer mit Azithromycin im Vergleich zu anderen Antibiotika deutlich verkürzt.

\section{Ketolide}

Eine weitere viel versprechende Alternative zur ambulanten Behandlung der Chlamydien-Pneumonie stellt die Therapie mit Ketoliden dar. Dies sind neu entwickelte Substanzen, die den Makroliden sowohl in ihrer Struktur als auch in ihrem Wirkspektrum ähneln, aber zum Teil auch noch gegen makrolidresistente Bakterien wirksam sind [104]. Telithromycin ist das erste im Handel befindliche Ketolid, welches als orale Applikationsform vorliegt. Es reichert sich nach nahrungsunabhängiger Resorption in der Bronchialschleimhaut, im Alveolarfilm der Lunge sowie in den Alveolarmakrophagen an, eignet sich damit besonders gut zur Therapie atypischer Pneumonieerreger. Eine Halbwertzeit von ca. 10 Stunden erlaubt die einmal tägliche Gabe von $800 \mathrm{mg}$ der Substanz. Die In-vitro-Aktivität des Telithromycins gegenüber Chlamydia pneumoniae ist mit der der neueren Makrolide Clarithromycin und Azithromycin vergleichbar $\left(\mathrm{MHK}_{90}\right.$ : $0,015-2 \mathrm{mg} / \mathrm{L}$ ) [105]. Daten zur bakteriologischen und klinischen Wirksamkeit von Telithromycin bei Vorliegen einer Chlamydien-Pneumonie stehen aber noch aus. Ein anderes Ketolid, das ABT773, befindet sich zurzeit noch in der klinischen Entwicklung. In-vitro-Untersuchungen weisen auf eine noch bessere Wirksamkeit gegenüber Chlamydia pneumoniae hin $\left(\mathrm{MHK}_{90}\right.$ : $0,008-0,015 \mathrm{mg} / \mathrm{L}$ ) [106].

\section{Fluorochinolone}

Als chlamydienwirksam gelten alle Fluorochinolone der Gruppen II, III und IV. Ausgezeichnete pharmakokinetische Eigenschaften inklusive einer sehr guten Gewebegängigkeit garantieren eine optimale Erreichbarkeit der intrazellulären Erreger. Hauptangriffspunkt aller Chinolone ist die chlamydiale DNS-Gyrase [107].

Die älteren Chinolone wie Ofloxacin oder Ciprofloxacin (Gruppe II) sind mit MHK-Werten zwischen 1 und $16 \mathrm{mg} / \mathrm{L}$ in vitro deutlich weniger aktiv gegen Chlamydia pneumoniae als die Makrolide und Tetracycline $[91,108]$. Neuere Substanzen wie Levofloxacin, Trovafloxacin und Moxifloxacin besitzen niedrigere MHKWerte $(0,25-1 \mathrm{mg} / \mathrm{L})$ und damit eine höhere In-vitro-Aktivität als die Vertreter der Gruppe II [91 - 96,98,109,110]. Sparfloxacin, Grepafloxacin, Gatifloxacin und Gemifloxacin sind zwar mit MHK-Werten von 0,03-0,5 mg/L am potentesten gegen Chlamydia pneumoniae in der Zellkultur wirksam, aber auch ihre MHK- 
Werte liegen noch immer über denen der Makrolid-Antibiotika und Tetracycline $[91,96,97,99-101,111,112]$.

Die klinische Chinolon-Wirksamkeit bei der Behandlung einer Chlamydia pneumoniae-Pneumonie wurde in einigen wenigen Studien zumeist retrospektiv untersucht. So zeigten Plouffe u. Mitarb. 1996 bei 83\% der mit Ofloxacin behandelten Patienten mit einer ambulant erworbenen Pneumonie durch Chlamydia pneumoniae ein klinisches Ansprechen auf die Therapie im Vergleich zu 75\% der nach herkömmlichem Schema ( $\beta$-Laktam plus Makrolid oder Tetracyclin) behandelten Patienten [113]. Ähnliche Ergebnisse erbrachte auch eine Multizenterstudie von File u. Mitarb., welche 1997 publiziert wurde [114]. Hier galten $98 \%$ der mit Levofloxacin behandelten Patienten als geheilt im Vergleich zu 93\% der Patienten, die Ceftriaxon bzw. Cefuroxim plus einem Makrolid oder einem Tetracyclin als Therapie erhielten. Leider existieren zur Zeit keine Daten zur Eradikation von Chlamydia pneumoniae bei Patienten mit entsprechender Pneumonie nach Fluorochinolontherapie. Diese sind aber im Hinblick auf eine mögliche Persistenz der Erreger mit der Folge einer chronischen Infektion von größter Bedeutung. Hinweise für die Ausbildung einer Chinolon-Resistenz der Chlamydien unter Therapie auch nach wiederholter Applikation der Substanzen ergeben sich zum jetzigen Zeitpunkt nicht.

Für die Therapie der Chlamydia pneumoniae-Pneumonie sollten neuere den älteren Fluorochinolonen vorgezogen werden. Hierfür bieten sich vor allem Moxifloxacin und Levofloxacin an. Beide Substanzen liegen sowohl in der oralen als auch in der intravenösen Applikationsform vor. Empfohlen wird die einmal tägliche Gabe von $400 \mathrm{mg}$ Moxifloxacin oder die ein- bis zweimal tägliche Gabe von 500 mg Levofloxacin über 10 bis 14 Tage. Aktuell wird auch die einmal tägliche Gabe von $750 \mathrm{mg}$ Levofloxacin geprüft. Ob sich hierdurch ggf. die Therapiedauer verkürzt, bleibt abzuwarten. Die Tatsache des konzentrationsabhängigen bzw. zeitunabhängigen antibakteriellen Wirkeffektes von Fluorochinolonen lässt aber die Möglichkeit einer Therapieverkürzung erahnen. Gatifloxacin und Gemifloxacin befinden sich noch in Phase-III- bzw. -IV-Prüfungen, deren Ergebnisse hinsichtlich der Wirkungen und Nebenwirkungen abgewartet werden sollten.

\section{Tetracycline}

Die Tetracycline, insbesondere das Doxycyclin und das Minocyclin, galten bisher neben den Makroliden als Antibiotika der ersten Wahl bei der Behandlung von Chlamydieninfektionen. Die MHK-Werte beider Substanzen entsprechen annähernd den MHK-Werten der Makrolid-Antibiotika (Doxycyclin: 0,016-0,25 $\mathrm{mg} / \mathrm{L}$, Minocyclin: 0,015-0,06 mg/L) [91-99,101]. Trotz guter In-vitro-Wirksamkeit gegen Chlamydien ist ihr Einsatz aufgrund ihrer nicht unerheblichen Nebenwirkungen nur noch bedingt und dann zur gezielten Erregertherapie zu empfehlen. Ein anderes Problem stellt die weltweit zunehmende Tetracyclin-Resistenz bei den typischen Pneumonieerregern wie den Pneumokokken dar, welche häufig als Zweiterreger bei einer Mischinfektion mit Chlamydia pneumoniae auftreten. Die Gabe von Doxycyclin erfolgt einmal täglich in einer Dosierung von $200 \mathrm{mg}$ über 10 bis 14 Tage, die Gabe von Minocyclin zweimal täglich in einer Dosierung von $50 \mathrm{mg}$ über denselben Zeitraum.

\section{Andere Antibiotika}

Neben den oben genannten Antibiotikaklassen besitzen auch Rifampicin und das Streptogramin Quinopristin/Dalfopristin eine erwiesene antichlamydiale Wirkung. Beide Substanzen spielen aber für die Therapie der Chlamydia pneumoniae-Pneumonie keine Rolle.

Zusammenfassend wird bei Patienten mit einer im ambulanten Bereich behandelbaren Chlamydieninfektion die orale Gabe eines neueren Makrolids, evtl. eines Ketolids empfohlen. Bei notwendiger stationärer Therapie muss intravenös mit einem Makrolid oder einem neueren Fluorchinolon behandelt werden. Eine Kombinationstherapie ist bei der gezielten Therapie der Chlamydien-Pneumonie nicht notwendig. Die Empfehlungen zur Therapiedauer der C.-pneumoniae-Pneumonie in internationalen Richtlinien reichen von 10-14 Tagen, ohne dass hierzu kontrollierte Studien vorliegen $[87,89]$.

\section{Präventiv- und Bekämpfungsmaßnahmen}

Für Chlamydia-pneumoniae-Infektionen sind gezielte Präventivmaßnahmen nicht bekannt. Es wird aber empfohlen, den Kontakt zu chronisch kranken, älteren und immunsupprimierten Personen zu vermeiden, um eine Ansteckung zu verhindern. Die Chlamydia psittaci-Erkrankung von Vögeln in Beständen von Züchtern und im Handel stellt dagegen eine anzeigepflichtige Tierseuche dar, deren Bekämpfung tierseuchenrechtlich geregelt ist. Zur Prävention von Chlamydia psittaci-Infektionen sollte das Personal in befallenden Vogelbeständen neben der allgemeinen Schutzkleidung auch Mund- und Nasenschutzmasken tragen. Die Fäkalienentsorgung bei den betroffenen Vögeln sollte häufig und hygienisch in verschlossenen Behältern erfolgen. Bei anamnestisch bekanntem Kontakt zu Vögeln und Auftreten von unklarem Fieber sollte an das Vorliegen einer Ornithose gedacht und entsprechende Untersuchungen veranlasst werden, um im Fall einer Erkrankung frühzeitig eine Therapie einleiten zu können. Für Kontaktpersonen ornithoseerkrankter Patienten besteht keine Ansteckungsgefahr, da eine Übertragung von Mensch zu Mensch sehr selten beschrieben ist. Über ein gehäuftes Auftreten der Ornithose wie z. B. bei Risikogruppen wie Vogelbesitzern, Beschäftigten in Geflügelfarmen oder in der Geflügelschlachtung, Tierpflegern oder Tierärzten, muss das zuständige Gesundheitsamt informiert werden, damit in Zusammenarbeit mit den Veterinärbehörden die Ursachen abgeklärt und Schutzmaßnahmen für die Beschäftigten und Maßnahmen zur Bekämpfung eines Ausbruchs eingeleitet werden können. Im Infektionsschutzgesetz ( $\S 7)$ ist festgelegt, dass der Erregernachweis von Chlamydia psittaci meldepflichtig ist, sofern der Verdacht auf eine akute Infektion besteht. 


\section{Literatur}

${ }^{1}$ Fang G, Fine M, Orloff $\mathrm{J}$ et al. New and emerging etiologies for community-acquired pneumonia with implications for therapy: a prospective multicenter study of 356 cases. Medicine 1990; 69: 307 - 316

${ }^{2}$ Mandall LA. Comunity-acquired pneumonia: etiology, epidemiology, and treatment. Chest 1995; (suppl) 108: 35-42

${ }^{3}$ Lode H, Steinhoff D, Schaberg T et al. New pneumonia pathogens, especially Chlamydia pneumoniae and Hantaviruses. Internist (Berl) 1996; 37: $882-889$

${ }^{4}$ Steinhoff D, Lode H, Ruckdeschel G et al. Chlamydia pneumoniae as a cause of community-acquired pneumonia is hospitalised patients in Berlin. Clin Infect Dis 1996; 22: 958 - 964

${ }^{5}$ Allewelt M, Steinhoff D, Rahlwes M et al. Wandel im Erregerspektrum ambulant erworbener Pneumonien (1982-1992). Dtsch Med Wschr 1997; 122: 1027-1032

${ }^{6}$ Plouffe JF. Importance of atypical pathogens of community-acquired pneumonia. Clin Infect Dis 2000; (suppl) 31: 35-39

${ }^{7}$ Cosentini R, Tarsia P, Blasi F et al. Community-acquired pneumonia: role of atypical organisms. Monalde Arch Chest Dis 2001; 56: $527-534$

${ }^{8}$ Gupta SK, Sarosi GA. The role of atypical pathogens in communityacquired pneumonia. Med Clin North Am 2001; 85: 1349-1365

${ }^{9}$ Marrie TJ. Community-acquired pneumonia. Clin Infect Dis 1994; 18 : $501-515$

${ }^{10}$ Bartlett JG, Mundy LM. Community-acquired pneumonia. New Engl J Med 1995; 333: $1618-1624$

${ }^{11}$ Kauppinen MT, Saikku P, Kujala P et al. Clinical picture of communityacquired Chlamydia pneumoniae pneumonia requiring hospital treatment: a comparison between chlamydial and pneumococcal pneumonia. Thorax 1996; 51: 185 - 189

12 Lieberman D, Schlaeffer F, Boldur I et al. Multiple pathogens in adult patients admitted with community-acquired pneumonia: a one year prospective study of 346 consecutive patients. Thorax 1996; 51: $179-184$

${ }^{13}$ Lieberman D, Ben-Yaakov M, Lazarovich Z et al. Chlamydia pneumoniae community-acquired pneumonia: a review of 62 hospitalized adult patients. Infection 1996; 24: 109-114

${ }^{14}$ Dowell SF, Peeling RW, Boman J et al. Standardizing Chlamydia pneumoniae assays: recommendations from the Centers for Disease Control and Prevention (USA) and the Laboratory Centre for Disease Control (Canada). Clin Infec Dis 2001; 33: $492-503$

${ }^{15}$ Kuo CC, Jackson LA, Campbell LA et al. Chlamydia pneumoniae (TWAR). Clin Microbiol Rev 1995; 8: 451 -461

${ }^{16}$ Schachter J, Stamm WE. Chlamydia. In: Murray PR, Baron EJ, Pfaller MA, Tenover FC, Yolken RH, eds. Manual of clinical microbiology. Washington, D.C.: 7th ed. Press, 1999: 669-677

17 Stephens RS(ed). Chlamydia: intracellular biology, pathogenesis, and immunity. Washington, D.C.: American Society for Microbiology, 1999

18 Everett KDE, Bush RM, Andersen AA. Emended description of the order Chlamydiales, proposal of Parachlamydiaceae fam. Nov. and Simkaniaceae fam. Nov., each containing one monotypic genus, revised taxonomy of the family Chlamydiaceae, including a new genus and five new species, and standards for the identifications of organisms. Int J Syst Bactiriol 1999; 49: 415-440

${ }^{19}$ Amann R, Springer N, Schönhuber W et al. Obligate intracellular bacterial parasites of acanthamoebae related to Chlamydia spp. Appl Environ Microbiol 1997; 63: 115-121

${ }^{20}$ Fritsche TR, Horn M, Wagner M et al. Phylogenetic diversity among geographically dispersed Chlamydiales endosymbionts recovered from clinical and environmental isolates of Acanthamoeba spp. Appl Environ Microbiol 2000; 66: 2613-2619

${ }^{21}$ Horn M, Wagner M, Müller KD et al. Neochlamydia hartmannellae gen. nov., sp. nov. (Parachlamydiaceae), an endoparasite of the amoeba Hartmanellae vermiformis. Microbiology 2000; 146: 1231 - 1239

${ }^{22}$ Kahane S, Everett KD, Kimmel N et al. Simkania negevensis strain ZT: growth, antigenic and genome characteristics. Int J Syst Bacteriol 1999; 49: 815-820

${ }^{23}$ Grayston JT, Diwan VK, Cooney M et al. Community- and hospitalacquired pneumonia associated with Chlamydia TWAR infection demonstrated serologically. Arch Intern Med 1989; 149: 169-173
${ }^{24}$ Grayston JT, Campbell LA, Kuo CC et al. A new respiratory tract pathogen: Chlamydia pneumoniae strain TWAR. J Infect Dis 1990; 161: $618-625$

${ }^{25}$ Chirgwin K, Roblin PM, Gelling M et al. Infection with Chlamydia pneumoniae in Brooklyn. J Infect Dis 1991; 163: $757-761$

${ }^{26}$ Kauppinen M, Saikku P. Pneumonia due to Chlamydia pneumoniae: prevalence, clinical features, diagnosis, and treatment. Clin Inf Dis 1995; (suppl) 3: 244-252

${ }^{27}$ Suttithawil W, Wangroongsarb P, Naigowit P et al. Chlamydia (Chlamydia) pneumoniae as a cause of community-acquired pneumonia in Thailand. J Med Assoc Thai 2001; 84: 430-437

${ }^{28}$ Kese D, Cizman M, Marin J et al. Chlamydia pneumoniae infections in patients with community-acquired pneumonia in Slovenia. Scand J Inf Dis 2002; 34: $172-176$

${ }^{29}$ Miyashita N, Fukano H, Okimoto N et al. Clinical presentation of community-acquired pneumonia in adults. Chest 2002; 121: 1776-1781

${ }^{30}$ Hammerschlag MR. Chlamydia pneumoniae and the lung. Eur Respir J 2000; 16: 1001 - 1007

31 Beaty CD, Grayston JC, Wang SP et al. Chlamydia pneumoniae, strain TWAR, infection in patients with chronic obstructive pulmonary disease. Am Rev Respir Dis 1991; 144: 1408-1410

32 Karnak D, Beder S. Treatment of Chlamydia pneumoniae infection and chronic obstructive pulmonary disease. Expert Opin Pharmacother 2002; 3: $1461-1470$

${ }^{33} \mathrm{Kraft}$ M. The role of bacterial infections in asthma. Clin Chest Med 2000; 21: $301-313$

${ }^{34}$ Hertzen L von. Role of persitent infection in the control and severity of asthma: focus on Chlamydia pneumoniae. Eur Respir J 2001; 19: $546-556$

${ }^{35}$ Wong YK, Gallagher PJ, Ward ME. Chlamydia pneumoniae and atherosclerosis. Heart 1999; 81: 232-238

${ }^{36}$ Campball LA, Kuo CC. Chlamydia pneumoniae pathogenesis. J Med Microbiol 2002; 51: 623-625

${ }^{37}$ Kalayoglu MV, Libby P, Byrne GI. Chlamydia pneumoniae as an emerging risk factor in cardiovascular diseases. JAMA 2002; 288: $2724-2731$

38 Ngeh J, Anand V, Gupta S. Chlamydia pneumoniae and atherosklerosis - what we know and what we don't. Clin Microbiol Infect 2002; 8: $2-13$

${ }^{39}$ Balin BJ, Appelt DM. Role of infection in Alzheimer' disease. J Am Osteopath Assoc 2001; (suppl) 12: 1-6

${ }^{40}$ Yucesan C, Sriram S. Chlamydia pneumoniae infection of the central nervous system. Curr Opin Neurol 2001; 14: 355-359

${ }^{41}$ Saikku P, Wang SP, Kleemola M et al. An epidemic of mild pneumonia due to an unusual strain of Chlamydia psittaci. J Infect Dis 1985; 151: $832-839$

${ }^{42}$ Storey C, Lusher M, Yates P et al. Evidence for Chlamydia pneumoniae of non-human origin. J Gen Bacteriol 1993; 139: 2621 - 2626

${ }^{43}$ Forsey T, Darougar S, Treharne JD. Prevalence in human beings of antibodies to Chlamydia IOL-207, an atypical strain of chlamydia. J Infect 1986; $12: 145-152$

${ }^{44}$ Kanamoto Y, Ouchi K, Mizui M et al. Prevalence of antibody to Chlamydia pneumoniae TWAR in Japan. J Clin Microbiol 1991; 29: $816-818$

${ }^{45}$ Marton A, Karolyi A, Szalka A. Prevalence of Chlamydia pneumoniae antibodies in Hungary. Eur J Clin Microbiol Infect Dis 1992; 11: $139-142$

${ }^{46}$ Montes M, Cilla G. High prevalence of Chlamydia pneumoniae infection in children and young adults in Spain. Pediatr Infect Dis J 1992; 11: 972 - 973

${ }^{47}$ Grayston JT. Infections caused by Chlamydia pneumoniae strain TWAR. Clin Inf Dis 1992; 15: 757-763

${ }^{48}$ Grayston JT, Diwan VK, Cooney M et al. Community- and hospitalacquired pneumonia associated with Chlamydia TWAR infection demonstrated serologically. Arch Intern Med 1989; 149: 169-173

${ }^{49}$ Cosentini R, Blasi F, Rossi S et al. Chlamydia pneumoniae and severe community-acquired pneumonia. In: Orfila J, Byrne GI, Cherneskey MA, Grayston JT, et al. eds. Chlamydial infections - 1994. Bologna, Italy: Societa Editrice Esculapio, 1994: 453-456

${ }^{50}$ Gaydos CA, Eiden JJ, Oldach D et al. Diagnosis of Chlamydia pneumoniae in patients with community-acquired pneumonia by polymerase chain reaction enzyme immunoassay. Clin Infect Dis 1994; 19: $157-160$

${ }^{51}$ Kauppinen M, Kujala P, Leinonen M et al. Clinical features of community-acquired Chlamydia pneumoniae pneumonia. In: Orfila J, Byrne 
GI, Cherneskey MA, et al. eds. Chlamydial infections - 1994. Bologna, Italy: Societa Editrice Esculapio, 1994: 457-460

${ }^{52}$ Wainwright AP, Beaumont AC, Kox WJ. Psittacosis: diagnosis and management of severe pneumonia and multi organ failure. Intensive Care Med 1987; 13: 419-421

${ }^{53}$ Gregory DW, Schaffner W. Psittacosis. Semin Respir Infect 1997; 12: 7- 11

${ }^{54}$ Elliott JH. Psittacosis. A flu like syndrome. Aust Fam Physician 2001; 30: $739-741$

${ }^{55}$ Cles LD, Stamm WE. Use of HL cells for improved isolation and passage of Chlamydia pneumoniae. J Clin Microbiol 1990; 28: 938-940

${ }^{56}$ Kuo CC, Grayston JT. A sensitive cell line, HL cells, for isolation and propagation of Chlamydia pneumoniae strain TWAR. J Inf Dis 1990; 162: $755-758$

${ }^{57}$ Wong KH, Skelton SK, Chan YK. Efficient culture of chlamydia pneumoniae with cell lines derived from the human respiratory tract. J Clin Microbiol 1992; 30: 1625 - 1630

${ }^{58}$ Grayston JT, Kuo CC, Wang SP et al. A new Chlamydia psittaci strain, TWAR, isolated in acute respiratory tract infection. N Engl J Med 1986; 315: $161-168$

${ }^{59}$ Kuo CC, Chen HH, Wang SP et al. Identification of a new group of Chlamydia psittaci strains called TWAR. J Clin Microbiol 1986; 24: $1034-1037$

${ }^{60}$ Person K, Haidl S. Evaluation of a commercial test for antibodies to the chlamydial lipopolysaccharide (Medac) for serodiagnosis of acute infections by Chlamydia pneumoniae (TWAR) and Chlamydia psittaci. APMIS 2000; 108: $131-138$

${ }^{61}$ Johnsen S, Andersen PL, Stanek G et al. Chlamydia antibody response in healthy volunteers immunized with nonchlamydial antigens: a randomized, double-blind, placebo-controlled study. Clin Inf Dis 2003; 36: 586-591

${ }^{62}$ Nurminen M, Leinonen M, Saikku P et al. The genus-specific antigen of Chlamydia: resemblance to the lipopolysaccharide of enteric bacteria. Science 1983; 220: 1279-1281

63 Peeling R. Chlamydia pneumoniae infections: applications of laboratory methods. In: Allegra L, Blasi F, eds. Chlamydia pneumoniae: the lung and the heart. Milano: Springer-Verlag Italia, 1999: 33-42

${ }^{64}$ Wang S. Serology for Chlamydia pneumoniae (TWAR). In: Allegra L, Blasi F, eds. Chlamydia pneumoniae: the lung and the heart. Milano: Springer-Verlag Italia, 1999: 16-23

${ }^{65}$ Wang SP, Grayston JT. Immunologic relationship between genital TRIC, lymphogranuloma venereum, and related organisms in a new microtiter indirect immunofluorescence test. Am J Ophthal 1970; 70: $367-374$

${ }^{66}$ Schachter J. Chlamydiae. In: Rose NR, DeMarcario EC, Fahey JL, Friedman H, Pen GM, eds. Manual of clinical laboratory immunology. Washington, D.C.: 4th ed. American Society for Microbiology, 1992: 661-666

${ }^{67}$ Ekman MR, Leinonen M, Syrjala $\mathrm{H}$ et al. Evaluation of serological methods in the diagnosis of Chlamydia pneumoniae pneumonia during an epidemic in Finland. Eur J Clin Microbiol Infect Dis 1993; 12: $756-760$

${ }^{68}$ Verkooyen RP, Lent NA van, Mousavi Joulandan SA et al. Diagnosis of Chlamydia pneumoniae in fection in patients with chronic obstructive pulmonary diseases by microimmunofluorescence and ELISA. J Med Microbiol 1997; 46: 959-964

${ }^{69}$ Verkooyen RP, Willemse D, Hiep-van Casteren SCAM et al. Evaluation of PCR, culture, and serology for diagnosis of Chlamydia pneumoniae respiratory infections. J Clin Microbiol 1998; 36: 2301 - 2307

${ }^{70}$ Peeling RW, Wang SP, Grayston JT et al. Chlamydia pneumoniae serology: interlaboratory variation in microimmunofluorescence assay results. J Inf Dis 2000; (suppl) 181: 426 - 429

${ }^{71}$ Messmer TO, Martinez J, Hassouna F et al. Comparison of two commercial microimmunofluorescence kits for detection of serum immunoglobulin G antibodies to Chlamydia pneumoniae. Clin Diagn Lab Immunol 2001; 8: 588-592

${ }^{72}$ Hermann C, Graf K, Groh A et al. Comparison of eleven commercial tests for Chlamydia pneumoniae-specific immunoglobulin $G$ in asymptomatic healthy individuals. J Clin Microbiol 2002; 40: $1603-1609$

73 Tugwell P, Dennis DT, Weinstein A et al. Laboratory evaluation in the diagnosis of Lyme disease. Ann Intern Med 1997; 127: 1109- 1123

${ }^{74}$ Bas S, Muzzin P, Ninet B et al. Chlamydial serology: comparative diagnostic value of immunoblotting, microimmunofluorescence test, and immunoassays using different recombinant proteins as antigens. J Clin Microbiol 2001; 39: 1368 - 1377

${ }^{75}$ Boman J, Allard A, Persson K et al. Rapid diagnosis of respiratory Chlamydia pneumoniae infection by nested touchdown polymerase chain reaction compared with culture and antigen detection by EIA.J Infect Dis 1997; 175: $1523-1526$

${ }^{76}$ Tong C, Sillis M. Detection of Chlamydia pneumoniae and Chlamydia psittaci in sputum samples by PCR. J Clin Pathol 1993; 46: 313 - 317

77 Campbell LA, Melgosa MP, Hamilton DJ et al. Detection of Chlamydia pneumoniae by polymerase chain reaction. J Clin Microbiol 1992; 30: 434-439

${ }^{78}$ Gaydos CA, Roblin PM, Hammerschlag MR et al. Diagnostic utility of PCR-enzyme immunoassay, culture and serology for detection of Chlamydia pneumoniae in symptomatic and asymptomatic patients. J Clin Microbiol 1994; 32: $903-905$

${ }^{79}$ Hyman CL, Roblin PM, Gaydos CA et al. Prevalence of asymptomatic nasopharyngeal carriage of Chlamydia pneumoniae in subjectively healthy adults: assessment by polymerase chain reaction-enzyme immunoassay and culture. Clin Infect Dis 1995; 20: 1174-1178

${ }^{80}$ Mygind T, Birkelund S, Birkebaek N et al. Determination of PCR efficiency in chelex-100 purified clinic samples and comparison of realtime quantitative PCR and conventional PCR for detection of Chlamydia pneumoniae. BMC Microbiol 2002; 9: 17

81 Tondella ML, Talkington DF, Holloway BP et al. Development and evaluation of real-time PCR-based fluorescence assays for detection of Chlamydia pneumoniae. J Clin Microbiol 2002; 40: 575-583

82 Kuoppa Y, Boman J, Scott L et al. Quantitative detection of respiratory Chlamydia pneumoniae infection by real-time PCR. J Clin Microbiol 2002; 40: 2273-2274

${ }^{83}$ Apfalter P, Barousch W, Nehr M et al. Comparison of a new quantitative ompA-based real-time PCR TaqMan assay for detection of Chlamydia pneumoniae DNA in respiratory specimens with four conventional PCR assays. J Clin Microbiol 2003; 41: 592-600

${ }^{84}$ Reischl U, Lehne N, Simnacher U et al. Rapid and standized detection of Chlamydia pneumoniae using LightCycler real-time fluorescence PCR. Eur J Clin Microbiol Infect Dis 2003; 22: 54-57

85 Welti M, Jaton K, Altwegg M et al. Development of a multiplex realtime quantitative PCR assay to detect Chlamydia pneumoniae, Legionella pneumophila and Mycoplasma pneumoniae in respiratory tract secretions. Diagn Microbiol Infect Dis 2003; 45: 85-95

${ }^{86}$ Poppert S, Essig A, Marre $\mathrm{R}$ et al. Detection and differentiation of Chlamydiae by fluorescence in situ hybridization. Appl Environ Microbiol 2002; 68: 4081 - 4089

${ }^{87}$ Niedermann MS, Mandell LA, Anzueto A et al. Guidelines for the initial management of adults with community-acquired pneumonia: diagnosis, assessment of severity, initial antimicrobial therapy, and prevention. Am J Respir Crit Care Med 2001; 148: 1730- 1754

88 Schaberg T, Dalhoff K, Ewig S et al. Deutsche Gesellschaft für Pneumologie. Empfehlungen zur Therapie der ambulant erworbenen Pneumonie. Pneumologie 1998; 51: 450-462

89 Bartlett JG, Dowell FS, Mandell LA et al. Practice guidelines for the management of community acquired pneumonia in adults. Clin Inf Dis 2000; $31: 347-382$

${ }^{90}$ Mandall LA, Marrie TJ, Grossman RF et al. Canadian guidelines for the initial management of community-acquired pneumonia: an evidence-based update by the Canadian Infectious Diseases Society and the Canadian Thoracic Society. Clin Infect Dis 2000; 31: 383-421

${ }^{91}$ Hammerschlag MR. Antimicrobial susceptibility and therapy of infections caused Chlamydia pneumoniae. Antimicrob Agents Chemother 1994; 38: 1873 - 1878

92 Roblin PM, Kutlin A, Hammerschlag MR. In vitro activity of trovafloxacin against Chlamydia pneumoniae. Antimicrob Agents Chemother 1997; 41: $2033-2034$

${ }^{93}$ Roblin PM, Hammerschlag MR. In vitro activity of a new 8-methoxyquinolone, BAY 12-8039, against Chlamydia pneumoniae. Antimicrob Agents Chemother 1998; 42: 951 - 952

${ }^{94}$ Hammerschlag MR, Qumei KK, Roblin PM. In vitro activities of azithromycin, clarithromycin, L-ofloxacin and other antibiotics against Chlamydia pneumoniae. Antimicrob Agents Chemother 1992; 36: $1573-1574$

${ }^{95}$ Ridgway GL, Salman H, Robbins MJ et al. In-vitro activity of grepafloxacin against Chlamydia spp., Mycoplasma spp., Ureaplasma urealyticum and Legionella spp. J Antimicrob Chemother 1997; (suppl) 40: $31-34$ 
${ }^{96}$ Miyashita N, Niki Y, Kishimoto T et al. In vitro and in vivo activities of AM-1155, a new fluoroquinolone, against Chlamydia spp. Antimicrob Agents Chemother 1997; 41: 1331 - 1334

${ }^{97}$ Donati M, Rodriguez Fermepin M, Olmo A et al. Comparative in-vitro activity of moxifloxacin, minocycline and azithromycin against Chlamydia spp. J Antimicrob Chemother 1999; 43: 825-827

98 Kimura M, Kishimoto T, Niki Y et al. In vitro and in vivo antichlamydial activities of newly developed quinolone antimicrobial agents. Antimicrob Agents Chemother 1993; 37: 801 - 803

${ }^{99}$ Roblin PM, Kutlin A, Reznik T et al. Activity of grepafloxacin and other fluoroquinolones and newer macrolides against recent clinical isolates of Chlamydia pneumoniae. Int J Antimicrob Agents 1999; 12: $181-184$

${ }^{100}$ Roblin PM, Hammerschlag MR. In vitro activity of Gatifloxacin against Chlamydia trachomatis and Chlamydia pneumoniae. J Antimicrob Chemother 1999; 44: 549-551

${ }^{101}$ Roblin PM, Reznik T, Kutlin A et al. In vitro activities of gemifloxacin (SB-265 805, LB20304) against recent clinical isolates of Chlamydia pneumoniae. Antimicrob Agents Chemother 1999; 43: 2806-2807

102 Block S, Hedrick J, Hammerschlag MR et al. Mycoplasma pneumoniae and Chlamydia pneumoniae in pediatric community-acquired pneumonia; comparative efficacy and safety of clarithromycin vs erythromycin ethylsuccinate. Pediatr Infect Dis J 1995; 14: 471 - 477

103 Roblin PM, Hammerschlag MR. Microbiologic efficacy of azithromycin and susceptibilities to azithromycin of isolates of Chlamydia pneumoniae from adults and children with community-acquired peumonia. Antimicrob Agents Chemother 1998; 42: 194-196

104 Balfour JA, Figgitt DP. Telithromycin. Drugs 2001; 61: 815-829

105 Roblin PM, Hammerschlag MR. In vitro activity of a new ketolide antibiotic, HMR 3647, against Chlamydia pneumoniae. Antimicrob Agents Chemother 1998; 42: 1515-1516

${ }^{106}$ Strigl S, Robin PM, Reznik T et al. In vitro activity of ABT 773, a new ketolide antibiotic, against Chlamydia pneumoniae. Antimicrob Agents Chemother 2000; 44: 1112-1113

107 Dessus-Babus S, Bebear CM, Charron A et al. Sequencing of gyrase and topoisomerase IV quinolone-resistence-determining regions of Chla- mydia trachomatis and characterization of quinolon-resistent mutants obtained in vitro. Antimicrob Agents Chemother 1998; 42: $1474-2481$

${ }^{108}$ Hammerschlag MR. Activity of gemifloxacin and other new quinolones against Chlamydia pneumoniae: a review. J Antimicrob Chemother 2000; (suppl) 45: 35-39

${ }^{109}$ Hammerschlag MR, Hyman CL, Roblin PM. In vitro activities of five quinolones against Chlamydia pneumoniae. Antimicrob Agents Chemother 1992; 36: 682-683

110 Woodcock JM, Andrews JM, Boswell FJ et al. In vitro activity of BAY 12-8039, a new fluoroquinolone. Antimicrob Agents Chemother 1997; 41: $101-106$

111 Wise R, Andrews JM, Brenwald N. The in-vitro activity of OPC-17116, a new 5-methyl substituted quinolone. J Antimicrob Chemother 1993; 31: $497-504$

112 Roblin PM, Montalban G, Hammerschlag MR. In vitro activities of OPC-17116, a new quinolone; ofloxacin; and sparfloxacin against Chlamydia pneumoniae. Antimicrob Agents Chemother 1994; 38: $1402-1403$

113 Plouffe JF, Herbert MT, File jr TM et al. Ofloxacin versus standard therapy in treatment of community-acqired pneumonia requiring hospitalization. Pneumonia Study Group. Antimicrob Agents Chemother 1996; 40: $1175-1179$

${ }^{114}$ File jr TM, Segreti J, Dunbar L et al. A multicenter, randomized study comparing the efficacy and safety of intravenous and/or oral levofloxacin versus ceftriaxone and/or cefuroxime axetil in treatment of adults with community-acquired pneumonia. Antimicrob Agents Chemother 1997; 41: 1965 - 1972

\section{Bereits publizierte Beiträge zu dieser Serie:}

${ }^{1}$ Diagnostik und Therapie der Legionellen-Pneumonie. Pneumologie 2002; 56: 695-703

2 Diagnostik, Therapie und Prävention der Influenza (Virusgrippe). Pneumologie 2003; 57: 27-33

\section{Buchbesprechung}

\section{Denkanstöße 2003 - Ein Lesebuch aus Philosophie, Kultur und Wissenschaft Hausner A (Hrsg)}

München: Piper Verlag GmbH, 2002. 199 S., Paperback. - Originalausgabe; Preis: $€ 5,-($ Österreich $€ 5,20$ )

ISBN 3-492-23616-2

Der Jahresband 2003, der seit 1985 erfolgreiche „Denkanstöße“ gibt, eine Übersicht zentraler Texte aus dem Wissenschafts- und Literatur-Programm des Piper-Verlages.

Die Herausgeberin hat - nach Goethes bewährter Regel „Wer vieles bringt ..." - eine klug ausgewählte Palette von divergierenden Themen, Essays, Textauszügen und Postulaten in drei Gruppen gegliedert: 1. Einsichten und Erfahrungen aus dem kulturellen Leben; 2. Erkenntnisse und Berichte aus den Naturwissenschaften; 3 . Thesen und Untersuchungen aus der historischen Forschung.

Und so spannt sich der Bogen der weitgefächerten und facettenreichen Beiträge u.a. vom Philosophen Karl Popper („Zurück zu den Vorsokratikern“), dem Nonkonformisten Hans Küng („Die Frau im Christentum“) zum Violin-Genie (und Denker) Yehudi Menuhin; von den Nobelpreisträgern und Physikern Richard P. Feynman („Vom Vergnügen etwas herauszufinden“) und dem „kreativen Physiker Werner Heisenberg“ (von Ernst Peter Fi- scher) zum Gehirnforscher Ian Robertson („Das Gehirn und sein Potential“) bis zu Bart Bosko („Fuzzy-Logik für Anfänger“); dann vom „Rätselhaften Hethiter-Reich“ (Birgit Brandau) schließlich zum aktuellen (doch heute schon geflissentlich verschwiegenen) Norman G. Finkelstein („Die Holocaust-Industrie“) und einer Stellungnahme hierzu von Philipp Blom („Das Gedenken - ein Geschäft?“) sowie Gerald Messadié mit „2000 Jahre Antisemitismus".

Die einzelnen Beiträge stehen in keinem unmittelbaren Zusammenhang miteinander - ein Vorteil, denn sie können unabhängig ausgewählt und gelesen werden, helfen so der Erinnerung und lassen Zusammenhänge wieder erkennen.

Bei der heutigen Überflutung mit Fach- und anderer Literatur (in Wort, Druck, Bild und Ton), zu derem Studium auch ein ambitioner Leser (mit „Bildungsbedürfnis“) kaum Zeit findet, sind diese „Denkanstöße“ ein vorzüglicher „Digest“! Das Buch benötigt weder Lesepult noch Bildschirm und bietet sich schon vom Format her als anspruchsvolle „Bettlektüre“ an.

Ein Quellenverzeichnis und Kurzvorstellungen der Autoren runden diese Sammlung vielfältiger und interessanter Beiträge $a b$, die - je nach Ambitionen und Gusto - zur weiteren „Nachlese“ anregen. Der scharfen Kritik eines „Literaturpapstes“ bedarf der Leser nicht - er entscheidet selber souverän.

Heinz S. Fuchs, Bad Godesberg 